\title{
On the Versatility of Bracha's Byzantine Reliable Broadcast Algorithm
}

\author{
Michel Raynal \\ Univ Rennes, Irisa, INRIA, CNRS, 35042 Rennes Cedex, France, and \\ Department of Computing, Polytechnic University, Hong Kong
}

\begin{abstract}
G. Bracha presented in 1987 a simple and efficient reliable broadcast algorithm for $n$-process asynchronous message-passing systems, which tolerates up to $t<n / 3$ Byzantine processes. Following an idea recently introduced by Hirt, Kastrato and Liu-Zhang (OPODIS 2020), instead of considering the upper bound on the number of Byzantine processes $(t)$, the present short article considers two types of Byzantine behavior: the ones that can prevent the safety property from being satisfied, and the ones that can prevent the liveness property from being satisfied (a Byzantine process can exhibit only one or both types of failures). This Byzantine differentiated failure model is captured by two associated upper bounds denoted $t_{s}$ (for safety) and $t_{\ell}$ for liveness). The article shows that only the threshold values used in the predicates of Bracha's algorithm must be modified to obtain an algorithm that works with this differentiated Byzantine failure model.
\end{abstract}

Keywords: Algorithm, Asynchronous system, Byzantine process, Distributed computing, Faulttolerance, Liveness property, Message-passing, Reliable broadcast, Safety property, Upper bound.

\section{Computing model}

Asynchronous processes This paper consider the classical system model made up of a finite set of $n>$ 1 asynchronous sequential processes, namely the set $\left\{p_{1}, \ldots, p_{n}\right\}$. "Asynchronous" means that each process proceeds at its own speed, which can vary arbitrarily with time, and always remains unknown to the other processes.

Communication network The processes communicate by exchanging messages through an asynchronous reliable point-to-point network. "Point-to-point" means that there is a bi-directional communication channel between each pair of processes (hence, no process can impersonate another process). "Reliable" means that the network neither lose, duplicate, modify, nor create messages. "Asynchronous" means that, while a message that has been sent is eventually received by its destination process, there is no bound on message transfer delays.

A process $p_{i}$ sends a message to a process $p_{j}$ by invoking the primitive "send TYPE $(m)$ to $p_{j}$ ", where TYPE is the type of the message and $m$ its content. To simplify the presentation, it is assumed that a process can send messages to itself. A process receives a message by executing the primitive "receive()". The (non-atomic) macro-operation "broadcast TYPE $(m)$ " is a shortcut for "for $j \in\{1, \cdots, n\}$ do send $\operatorname{TYPE}(m)$ to $p_{j}$ end for". 
Failure model A Byzantine process is a process that behaves arbitrarily: it can crash, fail to send or receive messages, send arbitrary messages, start in an arbitrary state, perform arbitrary state transitions, etc. $[8,12]$. A process that exhibits a Byzantine behavior is also called faulty. A non-Byzantine process is called correct or non-faulty.

Byzantine processes can collude to "pollute" the computation. They can also control the network in the sense that they can re-order message deliveries at correct processes, and determine the message delay of each message (but neither modify its content nor discard it).

As in [6] we consider a differentiated process failure model that distinguishes two types of Byzantine behavior $^{1}$ : the ones that can prevent the safety property from being satisfied (e.g., sending fake values), and the ones that can prevent the liveness property from being satisfied (e.g., not sending or not receiving a message). The integer $t_{s}$ (resp., $t_{\ell}$ ) denotes the maximal number of processes beyond which the safety (resp., liveness) property cannot be ensured. More precisely, if a Byzantine process exhibits only one type of bad behavior, it counts for " 1 " in the corresponding bound $t_{s}$ or $t_{\ell}$. If it exhibits both types of bad behavior it is counted for " 1 " in both $t_{s}$ and $t_{\ell}$. In the case where each Byzantine exhibits both types of bad behavior we have $t_{s}=t_{\ell}=t$.

\section{Byzantine Reliable Broadcast}

Definition This communication abstraction (in short BRB) provides each process with two operations, denoted BRB_broadcast () and BRB_deliver () . As in [5, 13], we use the following terminology: when a process invokes BRB_broadcast(), we say that it "brb-broadcasts a message", and when it executes BRB_deliver(), we say that it "brb-delivers a message". BRB is defined by the following properties.

- BRB-Validity. If a correct process brb-delivers a message $m$ from a correct process $p_{i}$, then $p_{i}$ brb-broadcast $m$.

- BRB-Integrity. Given a process $p_{i}$, a correct process brb-delivers at most one message from $p_{i}$.

- BRB-Termination. If (i) a correct process $p_{i}$ brb-broadcasts a message $m$, or (ii) a correct process brb-delivers a message $m$ from a process $p_{j}$, then all the correct processes brb-deliver the message $m$.

On safety properties The safety properties are on the nature of the messages that are brb-delivered (hence, they are trivially satisfied if no message is ever delivered). BRB-validity relates outputs (messages brb-delivered) to inputs (the brb-broadcast messages). BRB-integrity states that there is no duplication.

On liveness properties The liveness properties are on the fact that messages must be brb-delivered. BRB-Termination (i) states that a message brb-broadcast by a correct process is brb-delivered by all correct processes. BRB-Termination (ii) gives its name to reliable broadcast. Whether $p_{j}$ is correct or not, every message brb-delivered from $p_{j}$ by a correct process is brb-delivered by all correct processes.

On agreement It follows from the BRB-Integrity property and the BRB-Termination (ii) property that all the correct processes BRB-deliver the same set of messages, and this set contains at least all the messages BRB-broadcast by the correct processes.

\footnotetext{
${ }^{1}$ Other approaches differentiating failure types have been proposed since a long time. An an example, the types dormant and arbitrary are introduced in [10], which correspond to omission/timing failures and Byzantine behaviors, respectively.
} 
On computability It is shown in [6] (with slightly different notations) that BRB can be solved in the differentiated Byzantine failure model, if and only if $n>2 t_{\ell}+t_{s}$. Hence, if $t_{\ell}=t_{s}=t$ this condition boils down to $n>3 t$, which is the bound in the non-differentiated Byzantine failure model $[1,2,13]$.

From a message to a sequence of messages BRB is a one-shot communication abstraction (a correct process invokes brb_broadcast() at most once). Hence, the BRB instance invoked by $p_{i}$ can be identified by the process index $i$.

A simple way to obtain a multi-shot BRB abstraction (M-BRB) consists in associating a unique tag (e.g., a sequence number) with each invocation of brb_broadcast(), so that a process $p_{i}$ now invokes brb_broadcast $\left(s n_{i}, m\right)$, where $s n_{i}$ is its current sequence number value. This M-MRB instance is then identified by the pair $\left\langle i, s n_{i}\right\rangle$. For the M-BRB abstraction, the BRB-Validity and BRB-Integrity properties become:

- M-BRB-Validity. If a correct process brb-delivers a message $m$ from a correct process $p_{i}$ with sequence number $s n$, then $p_{i}$ brb-broadcast $m$ with sequence number $s n$.

- M-BRB-Integrity. Given a sequence number $s n$ and a process $p_{i}$, a correct process brb-delivers at most one message $m$ associated with $s n$ from $p_{i}$.

the same as their BRB counterparts, with the addition that sequence numbers are also preserved.

On BRB algorithms Bracha's BRB algorithm [1] implements the BRB abstraction, with optimal $t$ resilience $(t<n / 3)$. Let an application message be a message sent by the BRB abstraction, and a protocol message be a message used to implement BRB. The BRB of an application message by a correct process requires 3 communication steps and $(n-1)(2 n+1)$ protocol messages.

It is natural that, as it is a fundamental communication abstraction, BRB has been addressed by many authors. Here are a few recent results.

- The BRB algorithm presented in [7], implements the brb-broadcast of an application message with only two communication steps, two message types, and $n^{2}-1$ protocol messages. The price to pay for this gain in efficiency is a weaker $t$-resilience, namely $t<n / 5$. Hence, this algorithm and Bracha's algorithm differ in the trade-off $t$-resilience versus message/time efficiency.

- Scalable BRB is addressed in [4]. The issue is here not to pay the $O\left(n^{2}\right)$ message complexity price. To this end, the authors use a non-trivial message-gossiping approach which allows them to design a sophisticated BRB algorithm satisfying fixed probability-dependent properties.

- A flexible Byzantine fault-tolerant approach is introduced in [9]. This new and very general approach relies on appropriate commit and BRB algorithms.

- BRB in dynamic systems is addressed [3]. Dynamic means that a process can enter and leave the system at any time. In their article the authors present an efficient BRB algorithm for such a context. This algorithm assumes that, at any time, the number of Byzantine processes present in the system is less than one third of total number of processes present in the system.

- An efficient algorithm for BRB with long inputs of $\ell$ bits using lower costs than $\ell$ single-bit instances is presented in [11]. This algorithm, which assumes $t<n / 3$, achieves the best possible communication complexity of $\Theta(n \ell)$ input sizes. This article also presents an authenticated extension of the previous algorithm. 


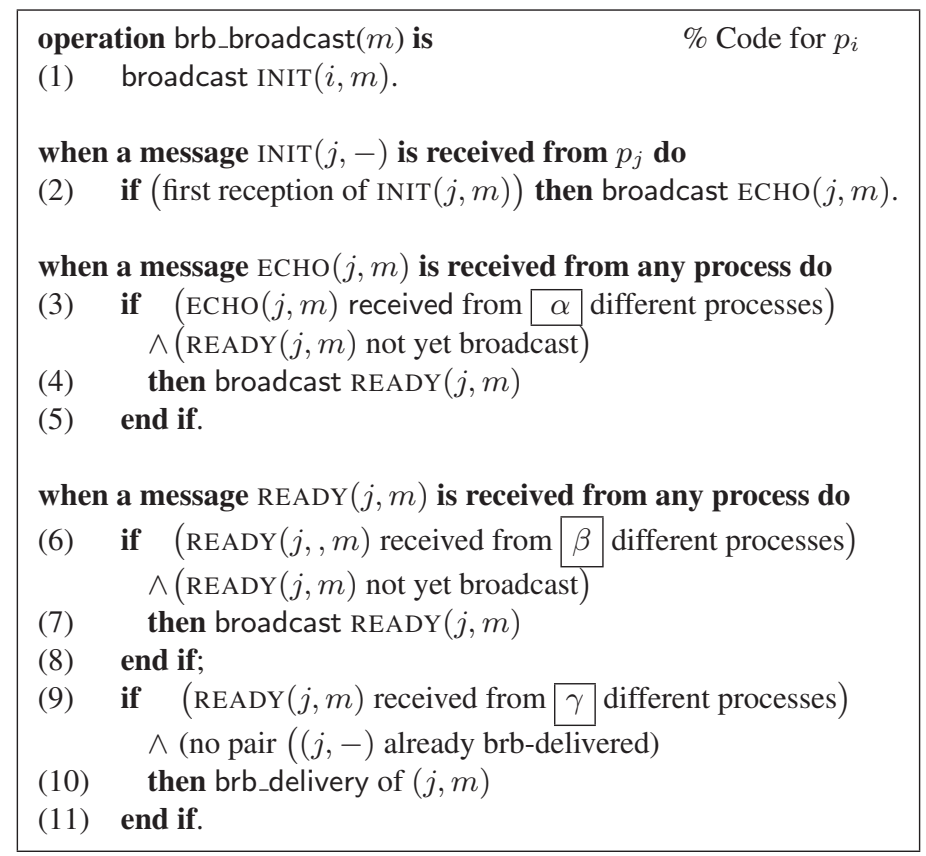

Figure 1: Skeleton of Bracha's BRB algorithm

\section{A Generic View of Bracha's Algorithm}

Skeleton of Bracha's algorithm The algorithm proposed by Bracha in [1] to implement BRB is based on a "double echo" mechanism of the message brb-broadcast by the sender process. The corresponding algorithm skeleton is described in Fig. 1, where the values $\alpha, \beta$ and $\gamma$ (which appear inside squares to make them more visible) are specific integer values.

When a process $p_{j}$ invokes brb_broadcast $(m)$ it sends the protocol message $\operatorname{INIT}(j, m)$ to all (line 1$)$. When a process $p_{i}$ receives a message $\operatorname{INIT}(j, m), p_{i}$ echoes it by sending to all the protocol message $\operatorname{ECHO}(j, m)$ (line 2). When $p_{i}$ has received "enough" $\operatorname{ECHO}(j, m)$ messages (namely $\alpha$ ), $p_{i}$ echoes it for the second time (message $\operatorname{READY}(j, m)$ sent at line 4). Then, when $p_{i}$ has received "enough" $\operatorname{READY}(j, m)$ messages (namely $\beta$ ), it participates to second echo if not yet done (lines 6-8). The aim of this second echo is to prevent a possible blocking. Finally, when $p_{i}$ has received "enough' $\operatorname{READY}(j, m)$ messages (namely $\gamma$ ), it brb-delivers the pair $(j, m)$.

It is easy to see that the BRB of an application message requires three types of protocol messages and three consecutive communication steps (one for each message type) which generates $(n-1)(2 n+1)$ protocol messages.

Three instances of Bracha's algorithm skeleton Table 1 presents three instantiations of Bracha's algorithm skeleton, each defined by specific values of the integers $\alpha, \beta$, and $\gamma$.

The second column corresponds to Bracha's algorithm which considers the bound $t$ on Byzantine processes, while the last two columns consider the pair of parameters $\left(t_{s}, t_{\ell}\right)$ as defined in the "Failure model" Section. The necessary and sufficient requirement $n>2 t_{s}+t_{\ell}$, which applies to the two rightmost columns, is proved in [6]. As we can see, the values of $\beta$ are the same in all the instantiations. Differently, the values of $\alpha$ and $\gamma$ are the same in the Bracha's column and the rightmost column, and smaller than the ones of the HKL's column as soon as $n \geq 3 t+x$ (for the Bracha's column) and $n \geq 2 t_{\ell}+t_{s}+x$ (for the HKL's column), for $x \geq 1$. Moreover, in some circumstances, the algorithm described in [6] requires one more communication step than the two other instantiations. Let us also notice that when $t_{s}=t_{\ell}=t$, the instantiation of the rightmost column boils down to Bracha's algorithm. 


\begin{tabular}{|c|c|c|c|}
\hline & Bracha [1] & HKL [6] & This paper \\
\hline \hline Requirement & $n>3 t$ & $n>2 t_{\ell}+t_{s}$ & $n>2 t_{\ell}+t_{s}$ \\
\hline \hline$\alpha$ & $\left\lfloor\frac{n+t}{2}\right\rfloor+1$ & $n-t_{\ell}$ & $\left\lfloor\frac{n+t_{s}}{2}\right\rfloor+1$ \\
\hline$\beta$ & $t+1$ & $t_{s}+1$ & $t_{s}+1$ \\
\hline$\gamma$ & $2 t+1$ & $n-t_{\ell}$ & $t_{s}+t_{\ell}+1$ \\
\hline Comm. steps & 3 & 3 or 4 & 3 \\
\hline
\end{tabular}

Table 1: Three instances of Bracha's generic skeleton

\section{Proof of the Third Instantiation}

The reader can find a proof of Bracha's algorithm in [1, 13], and the proof of Hirt, Kastrato, and LiuZhang's algorithm in [6]. So, only the proof of the last instantiation is presented here.

Preliminary remark In the following $f$ denotes the actual number of Byzantine processes in a run of the algorithm instantiated with $\alpha=\left\lfloor\frac{n+t_{s}}{2}\right\rfloor+1, \beta=t_{s}+1$, and $\gamma=t_{s}+t_{\ell}+1$ (rightmost column of the table). As any subset of the $f$ Byzantine processes can participate only in $t_{s}$ or only in $t_{\ell}$, and this is not known in advance (as in [6]), it is assumed that $f \leq t_{s}$ and $f \leq t_{\ell}$. Moreover, $n>2 t_{\ell}+t_{s}$.

Lemma 1. Let $f \leq t_{s}$ and $n>2 t_{\ell}+t_{s}$. If a correct process $p_{i}$ brb-delivers the message $m$ from a correct process $p_{j}$, then $p_{j}$ brb-broadcast $m$.

Proof As no correct process forges fake messages, the only cause for a correct process $p_{i}$ to brb-deliver a message $m$ from a correct process $p_{j}$ is because $p_{j}$ brb-broadcast $m$ or because the Byzantine processes forged messages that create the illusion that $m$ was brb-broadcast by $p_{j}$. We show that the second scenario is not possible.

To this end, let us assume by contradiction that $p_{j}$ never issued brb_broadcast $(m)$ and the pair $(j, m)$ has been forged by Byzantine processes. As there are at most $t_{s}$ Byzantine processes, they can generate at most $t_{s}$ fake messages $\operatorname{ECHO}(j, m)$, and $t_{s}$ fake messages $\operatorname{READY}(j, m)$. So a correct process can receive the fake message $\operatorname{ECHO}(j, m)$ from at most $t_{s}$ Byzantine processes and similarly for the fake message $\operatorname{READY}(j, m)$.

As $n>2 t_{\ell}+t_{s}$, and $\alpha=\left\lfloor\frac{n+t_{s}}{2}\right\rfloor+1$ we have $\alpha>t_{s}$. Hence, a correct process cannot send the message $\operatorname{READY}(j, m)$ at line 4 (because $\alpha>t_{s}$ ), and at line 7 (because $\beta>t_{s}$ ). Finally, as $\gamma>t_{s}$, the brb-delivery predicate of line 9 cannot be satisfied at a correct process $p_{i}$. It follows that, if a correct process $p_{i}$ brb-delivers the message $m$ from a correct process $p_{j}, p_{j}$ brb-broadcast $m . \quad \square$ Lemma 1

Lemma 2. A correct process brb-delivers at most one message $m$ from a process $p_{j}$.

Proof The proof follows directly from the brb-delivery predicate at line 9.

Lemma 3. Let $f \leq t_{\ell}$ and $n>2 t_{\ell}+t_{s}$. If a correct process brb-broadcasts a message $m$, all the correct processes brb-delivers $m$ from $p_{i}$.

Proof Let $p_{j}$ be a correct process that invokes brb_broadcast $(m)$. It follows that each correct process $p_{i}$ receives the message $\operatorname{INIT}(j, m)$ from $p_{j}$ and sends the message $\operatorname{ECHO}(j, m)$ to all the processes. Hence, each correct process receives the message $\operatorname{ECHO}(j, m)$ from $n-f$ correct processes. As $n-f \geq$ $n-t_{\ell} \geq\left\lfloor\frac{n+t_{s}}{2}\right\rfloor+1=\alpha$, each correct process sends $\operatorname{READY}(j, m)$ to all the processes (line 4$)$. Finally, 
as $n-f \geq n-t_{\ell} \geq t_{\ell}+t_{s}+1$, each correct process receives enough messages $\operatorname{READY}(j, m)$ that allows it to brb-deliver $m$.

Lemma 4. Let $f \leq t_{\ell}$. If a correct process brb-delivers a message $m$ from a process $p_{j}$, all the correct processes brb-delivers $m$ from $p_{j}$.

Proof Let $p_{i}$ be a process that brb-delivers $m$ from $p_{j}$. It follows from line 9 that $p_{i}$ received the message $\operatorname{READY}(j, m)$ from at least $\gamma=t_{\ell}+t_{s}+1$ different processes. We consider two cases.

- Case 1: $f \leq t_{\ell} \leq t_{s}$. As there are $f \leq t_{\ell}$ Byzantine processes, we can conclude from the value of $\gamma=t_{\ell}+t_{s}+1$ that at least $t_{s}+1$ correct processes sent the message $\operatorname{READY}(j, m)$. It then follows that each correct process receives the message $\operatorname{READY}(j, m)$ from $t_{s}+1$ correct processes, and (due to the predicate of line 6) sent this message to all the correct processes. It follows that each correct process receives the message $\operatorname{READY}(j, m)$ from $n-f \geq t_{\ell}+t_{s}+1$ different processes, which allows it to brb-deliver $m$ from $p_{j}$.

- Case 2: $f \leq t_{s}<t_{\ell}$. Exchanging $t_{s}$ and $t_{\ell}$, and observing that we have now $t s+1<t_{\ell}+1$, the reasoning is the same as in previous case ${ }^{2}$.

Theorem 1. Let $n>2 t_{\ell}+t_{s}$. Given a run, let $f$ be the number of Byzantine processes. If $f \leq t_{s}, t_{\ell}$, Algorithm 1 instantiated with $\alpha=\left\lfloor\frac{n+t_{s}}{2}\right\rfloor+1, \beta=t_{s}+1$, and $\gamma=t_{s}+t_{\ell}+1$, implements the BRB broadcast abstraction.

Proof The proof derives from the Lemmas 1 and 2 for the safety properties, and from the Lemmas 3 and 4 for the liveness properties.

$\square_{\text {Theorem } 1}$

\section{Conclusion}

Considering Bracha's Byzantine reliable broadcast (BRB) abstraction and the differentiated approach (as introduced in $[6,10]$ ), this article has shown that Bracha's algorithm is based on a powerful skeleton (made up of predicates associated with message exchange patterns), that has a versatility dimension which allows us to have different instantiations according to the thresholds associated with each message forwarding (echo mechanism). As shown by the last two columns of Table 1 it follows that the proposed instantiation of Bracha's BRB skeleton is more efficient that the one described in [6] (which may require four sequential communication phases instead of three).

As, due to its very definition, the behavior of a Byzantine process cannot be predicted, a practical approach consists in taking $t_{\ell}=t_{s}=t$. Nevertheless, the differentiated approach, that distinguishes the behaviors that prevent safety from the behaviors that prevent liveness is worth studying because it allows us to better understand the essence of algorithms implementing the BRB abstraction, which is one of the fundamental abstractions of fault-tolerant message-passing distributed computing.

\section{Acknowledgments}

The author want to thank the reviewers for their constructive comments, which help improve both the presentation and the content of the article.

\footnotetext{
${ }^{2}$ This comes from the fact that, in this lemma, $t_{s}$ and $t_{\ell}$ plays a symmetrical role, the important point being that $f \leq t_{s}$ and $f \leq t_{\ell}$.
} 
This work was partially supported by the French ANR Project (devoted to layered and modular structures in distributed computing) and ByBLoS (devoted to the design of building blocks for largescale trustless multi-users applications).

\section{References}

[1] Bracha G., Asynchronous Byzantine agreement protocols. Information \& Computation, 75(2):130-143 (1987)

[2] Bracha G. and Toueg S., Asynchronous consensus and broadcast protocols. Journal of the ACM, 32(4):824-840 (1985)

[3] Guerraoui G., Komatovic J., Kuznetsov P., Pignolet P.A., Seredinschi D.-A., and Tonkikh A., Dynamic Byzantine reliable broadcast. Proc. 24th Int'l Conference on Principles of Distributed Systems (OPODIS'20), LIPIcs Vol. 184, Article 23, 18 pages (2020)

[4] Guerraoui G., Kuznetsov P., Monti M., Pavlovic M., and Seredinschi D.-A., Scalable Byzantine reliable broadcast. Proc. $33 r d$ Int'l Symposium on Distributed Computing (DISC'19), LIPIcs Vol. 146, Article 22, 16 pages (2019)

[5] Hadzilacos V. and Toueg S., A modular approach to fault-tolerant broadcasts and related problems. Tech Report 94-1425, 83 pages, Cornell University, Ithaca (1994)

[6] Hirt M., Kastrato A., and Liu-Zhang C.-D., Multi-threshold asynchronous reliable broadcast and consensus. Proc. 24th Int'l Conference on Principles of Distributed Systems (OPODIS'20), LIPICs Vol. 184, Article 6, 16 pages (2020)

[7] Imbs D. and Raynal M., Trading t-resilience for efficiency in asynchronous Byzantine reliable broadcast. Parallel Processing Letters, Vol. 26(4), 8 pages (2016)

[8] Lamport L., Shostack R., and Pease M., The Byzantine generals problem. ACM Transactions on Programming Languages and Systems, 4(3)-382-401, (1982)

[9] Malkhi D., K Nayak K., and Ren L., Flexible Byzantine fault tolerance. Proc. ACM SIGSAC Conference on Computer and Communications Security (CCS'19), ACM Press, pp. 1041-1053 (2019)

[10] Meyer F.J. and Pradhan D.K., Consensus with dual failure modes. IEEE Transactions on Parallel and Distributed Systems, 2(2):214-222 (1991)

[11] Nayak K., Ren L., Shi E., Vaidya N.H., Xiang Z., Improved extension protocols for Byzantine broadcast and agreement. Proc. 34rd Int'l Symposium on Distributed Computing (DISC’20), LIPIcs Vol. 179, Article 28, 16 pages (2020)

[12] Pease M., Shostak R., and Lamport L., Reaching agreement in the presence of faults. Journal of the ACM, 27:228-234 (1980)

[13] Raynal M., Fault-tolerant message-passing distributed systems: an algorithmic approach. Springer, 480 pages, ISBN 978-3-319-94140-0 (2018) 